Article

\title{
Joint Transmit Antenna Selection and Power Allocation for ISDF Relaying Mobile-to-Mobile Sensor Networks
}

\author{
Lingwei $X u^{1, *}$, Hao Zhang ${ }^{1,2}$ and T. Aaron Gulliver ${ }^{2}$ \\ 1 College of Information Science and Engineering, Ocean University of China, Qingdao 266100, China; \\ gaomilaojia2009@163.com (L.X.); zhanghao@ccompass.com.cn (H.Z.) \\ 2 Department of Electrical and Computer Engineering, University of Victoria, Victoria, BC V8W 2Y2, Canada; \\ agullive@ece.uvic.ca (T.A.G.) \\ * Correspondence: gaomilaojia2009@163.com; Tel.: +86-182-5324-8508; Fax.: +86-532-6678-2926 \\ Academic Editor: Leonhard Reindl \\ Received: 5 January 2016; Accepted: 14 February 2016; Published: 19 February 2016
}

\begin{abstract}
The outage probability (OP) performance of multiple-relay incremental-selective decode-and-forward (ISDF) relaying mobile-to-mobile (M2M) sensor networks with transmit antenna selection (TAS) over N-Nakagami fading channels is investigated. Exact closed-form OP expressions for both optimal and suboptimal TAS schemes are derived. The power allocation problem is formulated to determine the optimal division of transmit power between the broadcast and relay phases. The OP performance under different conditions is evaluated via numerical simulation to verify the analysis. These results show that the optimal TAS scheme has better OP performance than the suboptimal scheme. Further, the power allocation parameter has a significant influence on the OP performance.
\end{abstract}

Keywords: M2M communications; N-Nakagami fading channels; incremental-selective decode-and-forward; outage probability; transmit antenna selection; power allocation

\section{Introduction}

To meet the increasing demands for high-data-rate services, mobile-to-mobile (M2M) communications has attracted significant interest from both industry and academia [1]. The M2M system architecture is described in [2]. In M2M communication systems, mobile users can directly communicate with each other without using a base station. This requires half the resources of traditional cellular communications, and thus improves the spectral efficiency and reduces the traffic load of the core network [3]. M2M communications can also be used to increase the data rate, reduce energy costs, reduce transmission delays, and extend the coverage area. Due to these advantages, M2M communications is an excellent choice for inter-vehicular communications, mobile sensor networks, and mobile heterogeneous networks [4]. M2M technologies have been proposed for home network applications [5]. In contrast to conventional fixed-to-mobile (F2M) cellular systems, both the transmitter and receiver in M2M systems can be in motion. Further, they are equipped with low elevation antennas. Thus, the widely employed Rayleigh, Rician, and Nakagami fading channels are not applicable to M2M communications systems [6]. Experimental results and theoretical analysis have shown that M2M channels can be described by cascaded fading channels [7]. The cascaded Rayleigh (also named as $N$-Rayleigh), fading channel was presented in [8]. The $N$-Rayleigh fading channel with $N=2$, denoted the double-Rayleigh fading model, was considered in [9]. The N-Rayleigh fading channel 
was extended to the $N$-Nakagami fading channel in [10]. The $N$-Nakagami fading model with $N=2$ is called the double-Nakagami fading model [11].

M2M communications may generate interference to existing cellular networks. To provide reliable cellular communications, the M2M transmit power and the distance between pairs of M2M users should be constrained. An M2M pair assisted by a relay can extend the coverage area with less transmit power. Therefore, relay-assisted M2M cooperative communications is an attractive solution to the interference problem. The pairwise error probability (PEP) of two relay-assisted vehicular scenarios using fixed-gain amplify-and-forward (FAF) relaying over double-Nakagami fading channels has been obtained [12]. An approximation for the average symbol error probability (SEP) has been derived for multiple-mobile-relay-based FAF relaying M2M cooperative networks over $N$-Nakagami fading channels [13]. Using the moment-generating function (MGF) approach, exact average SEP expressions for an AF M2M system over N-Nakagami fading channels have been derived [14].

Because of their low complexity, selective relaying (SR) and incremental relaying (IR) are widely employed in cooperative networks. Closed-form expressions for the error probability of incremental DF (IDF) and incremental AF (IAF) relaying over Rayleigh fading channels have been derived [15]. Further, an opportunistic IDF cooperation scheme employing orthogonal space-time block codes (OSTBC) over Rayleigh fading channels has been proposed [16]. In [17], closed-form OP expressions for IAF relaying M2M cooperative networks over $N$-Nakagami fading channels were derived. Exact average bit error probability (BEP) expressions for IDF relaying M2M cooperative networks over $\mathrm{N}$-Nakagami fading channels were derived in [18].

Selective relaying (SR) cooperative networks are not efficient in terms of time and frequency resources. In IR cooperative networks, if the signal-to-noise ratio (SNR) of the link between the source and relay is low, messages may not be decoded correctly by the relay, which can cause error propagation. Considering these problems, Chen et al. [19] proposed a novel incremental-selective DF (ISDF) relaying scheme over Rayleigh fading channels which combines the IDF and SDF relaying protocols [19]. Exact closed-form OP expressions for ISDF relaying M2M cooperative networks over $\mathrm{N}$-Nakagami fading channels were derived in [20].

Multiple-input-multiple-output (MIMO) transmission is a powerful technique which can be used to enhance the reliability and capacity of wireless systems. However, MIMO systems require multiple radio frequency chains, which increases the hardware complexity of the system. Transmit antenna selection (TAS) has been proposed as a practical way to reduce this complexity. In [21], the performance of optimal and suboptimal TAS schemes was investigated. Closed-form OP expressions for TAS MIMO networks over fading channels were derived in [22], and the energy efficiency was evaluated in [23].

Optimum power allocation is an important consideration in realizing the full potential of relay-assisted transmission. The resource allocation problem in both the uplink and downlink of two-tier networks comprising spectrum-sharing femtocells and macrocells has been investigated [24]. Further, the joint uplink subchannel and power allocation problem in cognitive small cells using cooperative Nash bargaining game theory was considered in [25]. A resource allocation scheme for orthogonal frequency division multiple access (OFDMA) based cognitive femtocells has been proposed [26], and secure resource allocation for OFDMA two-way relay wireless sensor networks was considered in [27].

However, to the best of our knowledge, the OP performance of ISDF relaying M2M sensor networks with TAS over $\mathrm{N}$-Nakagami fading channels has not been investigated. Moreover, most results in the literature do not consider the power allocation. This is important, as it can have a significant effect on the OP performance. The main contributions of this paper are as follows:

1. Closed-form expressions for the probability density function (PDF) and cumulative density functions (CDF) of the SNR over N-Nakagami fading channels are presented. These are used to derive exact closed-form OP expressions for optimal and suboptimal TAS schemes. These expressions can be used to evaluate the performance of inter-vehicular networks, mobile wireless sensor networks, and mobile heterogeneous networks. 
2. The power allocation problem is formulated to determine the optimum power distribution between the broadcast and relay phases.

3. The accuracy of the analytical results under different conditions is verified through numerical simulation. Results are given which show that the optimal TAS scheme has better OP performance than the suboptimal scheme. It is further shown that the power allocation parameter has a significant influence on the OP performance.

4. The OP expressions presented can be used to evaluate the performance of senor communication systems employed in inter-vehicular networks, mobile wireless sensor networks and mobile heterogeneous networks.

The rest of this paper is organized as follows. The multiple-mobile-relay-based M2M sensor network model is presented in Section 2. Section 3 provides exact closed-form OP expressions for the optimal TAS scheme. Exact closed-form OP expressions for the suboptimal TAS scheme are given in Section 4. In Section 5, the OP is optimized based on the power allocation parameter. Monte Carlo simulation results are presented in Section 6 to verify the analytical results. Finally, some concluding remarks are given in Section 7.

\section{The System and Channel Model}

\subsection{System Model}

The cooperation model consists of a single mobile source (MS) sensor, $L$ mobile relay (MR) sensors, and a single mobile destination (MD) sensor, as shown in Figure 1. The nodes operate in half-duplex mode. The MS is equipped with $N_{t}$ antennas, the MD is equipped with $N_{r}$ antennas, and the MR is equipped with a single antenna.

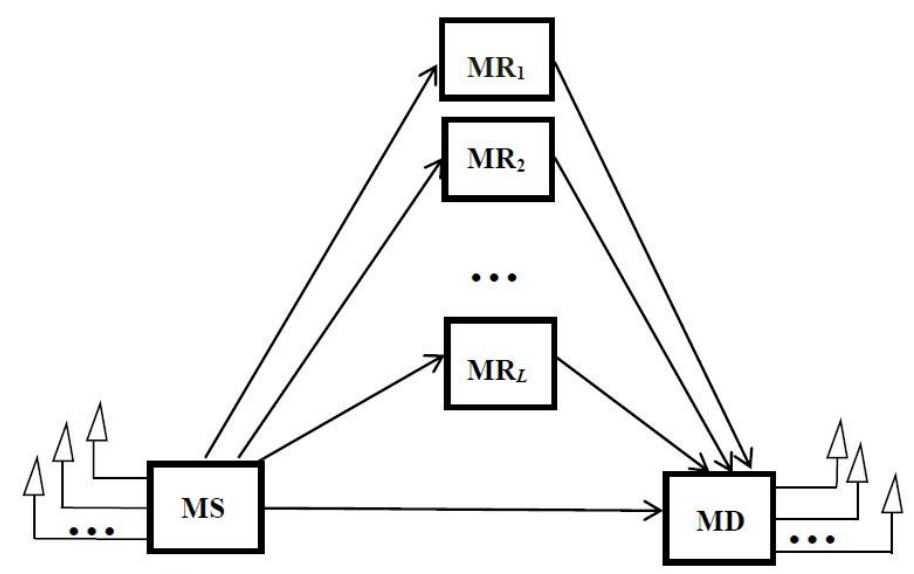

Figure 1. The system model.

It is assumed that the antennas at the MS and MD have the same distance to the relay nodes. Using the approach in [11], the relative gain of the MS to MD link is $G_{S D}=1$, the relative gain of the MS to $\mathrm{MR}_{l}$ link is $G_{\mathrm{SR} l}=\left(d_{\mathrm{SD}} / d_{\mathrm{SR} l}\right)^{v}$, and the relative gain of the $\mathrm{MR}_{l}$ to $\mathrm{MD}$ link is $\mathrm{G}_{\mathrm{RD} l}=\left(d_{\mathrm{SD}} / d_{\mathrm{RD} l}\right)^{v}$, where $v$ is the path loss coefficient, and $d_{\mathrm{SD}}, d_{\mathrm{SR} l}$, and $d_{\mathrm{RD} l}$ are the distances of the MS to MD, MS to $\mathrm{MR}_{l}$, and $\mathrm{MR}_{l}$ to $\mathrm{MD}$ links, respectively [28]. To indicate the location of $\mathrm{MR}_{l}$ with respect to the MS and $\mathrm{MD}$, the relative geometrical gain $\mu_{l}=G_{\mathrm{SR} l} / G_{\mathrm{RD} l}$ is defined. When $\mathrm{MR}_{l}$ is closer to the MD, $\mu_{l}$ is less than 1 , and when $\mathrm{MR}_{l}$ is closer to the MS, $\mu_{l}$ is greater than 1 . When $\mathrm{MR}_{l}$ has the same distance to the MS and MD, $\mu_{l}$ is $1(0 \mathrm{~dB})$.

Let $\mathrm{MS}_{i}$ denote the $i$ th transmit antenna at $\mathrm{MS}$ and $\mathrm{MD}_{j}$ denote the $j$ th receive antenna at MD. Further, let $h=h_{k}, k \in\left\{\mathrm{SD}_{i j}, \mathrm{SR}_{i l}, \mathrm{RD}_{l j}\right\}$ represent the complex channel coefficients of the $\mathrm{MS}_{i}$ to $\mathrm{MD}_{j}$, 
$\mathrm{MS}_{i}$ to $\mathrm{MR}_{l}$, and $\mathrm{MR}_{l}$ to $\mathrm{MD}_{j}$ links, respectively. If the $i$ th antenna at the MS is selected, during the first time slot the received signal $r_{\mathrm{SD} i j}$ at $\mathrm{MD}_{j}$ is given by

$$
r_{\mathrm{SD} i j}=\sqrt{K E} h_{\mathrm{SD} i j} x+n_{\mathrm{SD} i j}
$$

and the received signal $r_{\mathrm{SR} i l}$ at $\mathrm{MR}_{l}$ by

$$
r_{\mathrm{SR} i l}=\sqrt{G_{\mathrm{SR} i l} K E} h_{\mathrm{SR} i l} x+n_{\mathrm{SR} i l}
$$

where $x$ denotes the transmitted symbol, and $n_{\mathrm{SR} i l}$ and $n_{\mathrm{SD} i j}$ are additive white Gaussian noise (AWGN) with zero mean and variance $N_{0} / 2$. During the two time slots, $E$ is the total energy used by the MS and $\mathrm{MR}$, and $K$ is the power allocation parameter.

During the second time slot, by comparing the instantaneous SNR $\gamma_{\mathrm{SD} i j}$ to a threshold $\gamma_{\mathrm{P}}$, only the best MR decides whether to be active.

If $\gamma_{\mathrm{SD} i j}>\gamma_{\mathrm{P}}$, then $\mathrm{MS}_{i}$ and the best MR will receive a 'success' message. $\mathrm{MS}_{i}$ then transmits the next message, and the best MR remains silent. The corresponding received $\mathrm{SNR}$ at $\mathrm{MD}_{j}$ is

$$
\gamma_{0 i j}=\gamma_{\mathrm{SD} i j}
$$

where

$$
\gamma_{\mathrm{SD} i j}=\frac{K\left|h_{\mathrm{SDij}}\right|^{2} E}{N_{0}}=K\left|h_{\mathrm{SD} i j}\right|^{2} \bar{\gamma}
$$

If $\gamma_{\mathrm{SD} i j}<\gamma_{\mathrm{P}}$, then $\mathrm{MS}_{i}$ and the best MR will receive a 'failure' message. By comparing the instantaneous SNR $\gamma_{\mathrm{SR} i}$ to a threshold $\gamma_{\mathrm{T}}$, the best MR decides whether to decode and forward the signal to the $\mathrm{MD}_{j}$, where $\gamma_{\mathrm{SR} i}$ represents the SNR of the link between $\mathrm{MS}_{i}$ and the best MR. The best MR is selected based on the following decision rule

$$
\gamma_{\mathrm{SR} i}=\max _{1 \leqslant l \leqslant L}\left(\gamma_{\mathrm{SR} i l}\right)
$$

where $\gamma_{\text {SRil }}$ represents the SNR of the $\mathrm{MS}_{i}$ to $\mathrm{MR}_{l}$ link, and

$$
\gamma_{\text {SR } i l}=\frac{K G_{\text {SR } i l}\left|h_{\text {SRil }}\right|^{2} E}{N_{0}}=K G_{\text {SR } i l}\left|h_{\text {SR } i l}\right|^{2} \bar{\gamma}
$$

If $\gamma_{\mathrm{Sr} i}<\gamma_{\mathrm{T}}$, then $\mathrm{MS}_{i}$ will transmit the next message, and the best MR will not be used for cooperation. The corresponding received $\mathrm{SNR}$ at $\mathrm{MD}_{j}$ is

$$
\gamma_{1 i j}=\gamma_{\mathrm{SD} i j}
$$

If $\gamma_{S r i}>\gamma_{T}$, then the best MR decodes and forwards the signal to $\mathrm{MD}_{j}$. The corresponding received signal at $\mathrm{MD}_{j}$ is

$$
r_{\mathrm{RD} j}=\sqrt{(1-K) G_{\mathrm{RD} j} E} h_{\mathrm{RD} j} x+n_{\mathrm{RD} j}
$$

where $n_{\mathrm{RD} j}$ is AWGN with zero mean and variance $N_{0} / 2$.

If $\mathrm{MD}_{j}$ uses selection combining (SC), the received $\mathrm{SNR}$ is given by

$$
\gamma_{\mathrm{SC} i j}=\max \left(\gamma_{\mathrm{SD} i j}, \gamma_{\mathrm{RD} j}\right)
$$

where $\gamma_{\mathrm{RD} j}$ represents the SNR of the link between the best $\mathrm{MR}$ and $\mathrm{MD}_{j}$. Using SC at the MD, the received SNR is

$$
\gamma_{\mathrm{SC}_{i}}=\max _{1 \leqslant j \leqslant N_{r}}\left(\gamma_{i j}\right)
$$


where

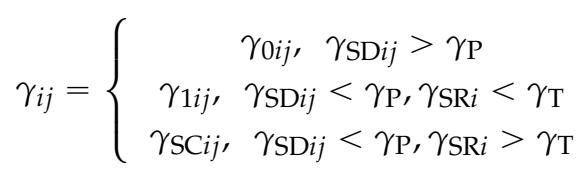

The optimal TAS scheme selects the transmit antenna $w$ that maximizes the received SNR at the MD, namely

$$
w=\max _{1 \leqslant i \leqslant N_{t}}\left(\gamma_{\mathrm{SC}_{i}}\right)=\max _{1 \leqslant i \leqslant N_{t}, 1 \leqslant j \leqslant N_{r}}\left(\gamma_{i j}\right)
$$

The suboptimal TAS scheme selects the transmit antenna $g$ that maximizes the instantaneous SNR of the direct link $\mathrm{MS}_{i}$ to $\mathrm{MD}_{j}$, namely

$$
g=\max _{1 \leqslant i \leqslant N_{t}, 1 \leqslant j \leqslant N_{r}}\left(\gamma_{\mathrm{SD} i j}\right)
$$

\subsection{System Model}

The links in the system are subject to independent and identically distributed N-Nakagami fading, so that $h$ follows the $N$-Nakagami distribution given by [10]

$$
h=\prod_{t=1}^{N} a_{t}
$$

where $N$ is the number of cascaded components, and $a_{t}$ is a Nakagami distributed random variable with PDF

$$
f(a)=\frac{2 m^{m}}{\Omega^{m} \Gamma(m)} a^{2 m-1} \exp \left(-\frac{m}{\Omega} a^{2}\right)
$$

$\Gamma(\cdot)$ is the Gamma function, $m$ is the fading coefficient, and $\Omega$ is a scaling factor.

Using the approach in [10], the PDF of $h$ is given by

$$
f(h)=\frac{2}{h \prod_{t=1}^{N} \Gamma\left(m_{t}\right)} G_{0, N}^{N, 0}\left[\left.h^{2} \prod_{t=1}^{N} \frac{m_{t}}{\Omega_{t}}\right|_{m_{1}, \ldots, m_{N}}\right]
$$

where $G[\cdot]$ is Meijer's G-function.

Let $y=\left|h_{k}\right|^{2}$ represent the square of the amplitude of $h_{k}$. The corresponding CDF and PDF of $y$ are [10]

$$
\begin{gathered}
F(y)=\frac{1}{\prod_{t=1}^{N} \Gamma\left(m_{t}\right)} G_{1, N+1}^{N, 1}\left[\left.y \prod_{t=1}^{N} \frac{m_{t}}{\Omega_{t}}\right|_{m_{1}, \ldots, m_{N}, 0} ^{1}\right] \\
f(y)=\frac{1}{y \prod_{t=1}^{N} \Gamma\left(m_{t}\right)} G_{0, N}^{N, 0}\left[\left.y \prod_{t=1}^{N} \frac{m_{t}}{\Omega_{t}}\right|_{m_{1}, \ldots, m_{N}} ^{-}\right]
\end{gathered}
$$

\section{The OP of the Optimal TAS Scheme}

The OP of the optimal TAS scheme can be expressed as

$$
F_{\text {optimal }}=\operatorname{Pr}\left(\max _{1 \leqslant i \leqslant N_{t}, 1 \leqslant j \leqslant N_{r}}\left(\gamma_{i j}\right)<\gamma_{\text {th }}\right)=\left(\operatorname{Pr}\left(\gamma_{i j}<\gamma_{\text {th }}\right)\right)^{N_{t} \times N_{r}}
$$


3.1. $\gamma_{t h}>\gamma_{P}$

If $\gamma_{\mathrm{th}}>\gamma_{\mathrm{P}}$, the OP of the optimal TAS scheme can be expressed as

$$
\left.\begin{array}{rl}
F_{\text {optimal }} & =\left(\begin{array}{c}
\operatorname{Pr}\left(\gamma_{\mathrm{p}}<\gamma_{\mathrm{SD}}, \gamma_{0}<\gamma_{\mathrm{th}}\right)+\operatorname{Pr}\left(\gamma_{\mathrm{SD}}<\gamma_{\mathrm{p}}, \gamma_{\mathrm{SR}}<\gamma_{\mathrm{T}}, \gamma_{1}<\gamma_{\mathrm{th}}\right) \\
+\operatorname{Pr}\left(\gamma_{\mathrm{SD}}<\gamma_{\mathrm{p}}, \gamma_{\mathrm{SR}}>\gamma_{\mathrm{T}}, \gamma_{\mathrm{SC}}<\gamma_{\mathrm{th}}\right)
\end{array}\right) \\
& =\left(G_{1}+G_{2}+G_{3}\right)^{N_{t} \times N_{r}}
\end{array}\right)^{N_{t} \times N_{r}}
$$

where $\gamma_{\text {th }}$ is the threshold for correct detection at the MD. $G_{1}$ is given by

$$
\begin{gathered}
G_{1}=\operatorname{Pr}\left(\gamma_{\mathrm{p}}<\gamma_{\mathrm{SD}}, \gamma_{0}<\gamma_{\mathrm{th}}\right)=\operatorname{Pr}\left(\gamma_{\mathrm{p}}<\gamma_{\mathrm{SD}}<\gamma_{\mathrm{th}}\right) \\
=\frac{1}{\prod_{d=1}^{N} \Gamma\left(m_{d}\right)}\left(G_{1, N+1}^{N, 1}\left[\left.\frac{\gamma_{\mathrm{th}}}{\bar{\gamma}_{\mathrm{SD}}} \prod_{d=1}^{N} \frac{m_{d}}{\Omega_{d}}\right|_{m_{1}, \ldots, m_{N}, 0}\right]-G_{1, N+1}^{N, 1}\left[\left.\frac{\gamma_{\mathrm{P}}}{\overline{\gamma_{\mathrm{SD}}}} \prod_{d=1}^{N} \frac{m_{d}}{\Omega_{d}}\right|_{m_{1}, \ldots, m_{N}, 0} ^{1}\right]\right) \\
\overline{\gamma_{\mathrm{SD}}}=K \bar{\gamma}
\end{gathered}
$$

$G_{2}$ can be written as

$$
\begin{aligned}
& G_{2}=\operatorname{Pr}\left(\gamma_{\mathrm{SD}}<\gamma_{\mathrm{p}}, \gamma_{\mathrm{SR}}<\gamma_{\mathrm{T}}, \gamma_{1}<\gamma_{\mathrm{th}}\right) \\
& =\operatorname{Pr}\left(\gamma_{\mathrm{SD}}<\gamma_{\mathrm{p}}, \gamma_{\mathrm{SR}}<\gamma_{\mathrm{T}}\right) \\
& =\frac{1}{\prod_{d=1}^{N} \Gamma\left(m_{d}\right)} G_{1, N+1}^{N, 1}\left[\left.\frac{\gamma_{\mathrm{P}}}{\overline{\gamma_{\mathrm{SD}}}} \prod_{d=1}^{N} \frac{m_{d}}{\Omega_{d}}\right|_{m_{1}, \ldots, m_{N}, 0} ^{1}\right] \times \\
& \left(\frac{1}{\prod_{t=1}^{N} \Gamma\left(m_{t}\right)} G_{1, N+1}^{N, 1}\left[\left.\frac{\gamma_{\mathrm{T}}}{\gamma_{\mathrm{SR}}} \prod_{t=1}^{N} \frac{m_{t}}{\Omega_{t}}\right|_{m_{1}, \ldots, m_{\mathrm{N}}, 0} ^{1}\right]\right)^{L} \\
& \overline{\gamma_{\mathrm{SR}}}=K G_{\mathrm{SR}} \bar{\gamma}
\end{aligned}
$$

and $G_{3}$ can be expressed as

$$
\begin{gathered}
G_{3}=\operatorname{Pr}\left(\gamma_{\mathrm{SD}}<\gamma_{\mathrm{p}}, \gamma_{\mathrm{SR}}>\gamma_{\mathrm{T}}, \gamma_{\mathrm{SC}}<\gamma_{\mathrm{th}}\right)=\operatorname{Pr}\left(\gamma_{\mathrm{SD}}<\gamma_{\mathrm{p}}, \gamma_{\mathrm{SR}}>\gamma_{\mathrm{T}}, \gamma_{\mathrm{RD}}<\gamma_{\mathrm{th}}\right) \\
=\frac{1}{\prod_{d=1}^{N} \Gamma\left(m_{d}\right)} G_{1, N+1}^{N, 1}\left[\left.\frac{\gamma_{\mathrm{P}}}{\overline{\gamma_{\mathrm{SD}}}} \prod_{d=1}^{N} \frac{m_{d}}{\Omega_{d}}\right|_{m_{1}, \ldots, m_{N}, 0} ^{1}\right] \times \frac{1}{\prod_{t t=1}^{N} \Gamma\left(m_{t t}\right)} G_{1, N+1}^{N, 1}\left[\left.\frac{\gamma_{\mathrm{th}}}{\overline{\gamma_{\mathrm{RD}}}} \prod_{t t=1}^{N} \frac{m_{t t}}{\Omega_{t t}}\right|_{m_{1}, \ldots, m_{N}, 0} ^{1}\right] \\
\times\left(1-\left(\frac{1}{\prod_{t=1}^{N} \Gamma\left(m_{t}\right)} G_{1, N+1}^{N, 1}\left[\left.\frac{\gamma_{\mathrm{T}}}{\overline{\gamma_{\mathrm{SR}}}} \prod_{t=1}^{N} \frac{m_{t}}{\Omega_{t}}\right|_{m_{1}, \ldots, m_{N}, 0} ^{1}\right]\right)\right) \\
\overline{\gamma_{\mathrm{RD}}}=(1-K) G_{\mathrm{RD}} \bar{\gamma}
\end{gathered}
$$

3.2. $\gamma_{t h}<\gamma_{P}$

If $\gamma_{\mathrm{th}}<\gamma_{\mathrm{P}}$, the OP of the optimal TAS scheme can be expressed as

$$
\begin{aligned}
F_{\text {optimal }} & =\left(\operatorname{Pr}\left(\gamma_{\mathrm{SD}}<\gamma_{\text {th }}, \gamma_{\mathrm{SR}}<\gamma_{\mathrm{T}}, \gamma_{1}<\gamma_{\text {th }}\right)+\operatorname{Pr}\left(\gamma_{\mathrm{SD}}<\gamma_{\text {th }}, \gamma_{\mathrm{SR}}>\gamma_{\mathrm{T}}, \gamma_{\mathrm{SC}}<\gamma_{\text {th }}\right)\right)^{N_{t} \times N_{r}} \\
& =\left(G_{11}+G_{22}\right)^{N_{t} \times N_{r}}
\end{aligned}
$$


where $G_{11}$ can be written as

$$
\begin{aligned}
& G_{11}=\operatorname{Pr}\left(\gamma_{\mathrm{SD}}<\gamma_{\mathrm{th}}, \gamma_{\mathrm{SR}}<\gamma_{\mathrm{T}}\right) \\
& =\frac{1}{\prod_{d=1}^{N} \Gamma\left(m_{d}\right)} G_{1, N+1}^{N, 1}\left[\left.\frac{\gamma_{\mathrm{th}}}{\gamma_{\mathrm{SD}}} \prod_{d=1}^{N} \frac{m_{d}}{\Omega_{d}}\right|_{m_{1}, \ldots, m_{N}, 0} ^{1}\right] \times\left(\frac{1}{\prod_{t=1}^{N} \Gamma\left(m_{t}\right)} G_{1, N+1}^{N, 1}\left[\left.\frac{\gamma_{\mathrm{T}}}{\gamma_{\mathrm{SR}}} \prod_{t=1}^{N} \frac{m_{t}}{\Omega_{t}}\right|_{m_{1}, \ldots, m_{N}, 0} ^{1}\right]\right)^{L}
\end{aligned}
$$

and $G_{22}$ is given by

$$
\begin{aligned}
& G_{22}=\operatorname{Pr}\left(\gamma_{\mathrm{SD}}<\gamma_{\mathrm{th}}, \gamma_{\mathrm{SR}}>\gamma_{\mathrm{T}}, \gamma_{\mathrm{RD}}<\gamma_{\mathrm{th}}\right) \\
& =\frac{1}{\prod_{d=1}^{N} \Gamma\left(m_{d}\right)} G_{1, N+1}^{N, 1}\left[\left.\frac{\gamma_{\mathrm{th}}}{\overline{\gamma_{\mathrm{SD}}}} \prod_{d=1}^{N} \frac{m_{d}}{\Omega_{d}}\right|_{m_{1}, \ldots, m_{N}, 0}\right] \times \frac{1}{\prod_{t t=1}^{N} \Gamma\left(m_{t t}\right)} G_{1, N+1}^{N, 1}\left[\left.\frac{\gamma_{\mathrm{th}}}{\overline{\gamma_{\mathrm{RD}}}} \prod_{t t=1}^{N} \frac{m_{t t}}{\Omega_{t t}}\right|_{m_{1}, \ldots, m_{N}, 0} ^{1}\right] \\
& \times\left(1-\left(\frac{1}{\prod_{t=1}^{N} \Gamma\left(m_{t}\right)} G_{1, N+1}^{N, 1}\left[\left.\frac{\gamma_{\mathrm{T}}}{\overline{\gamma_{\mathrm{SR}}}} \prod_{t=1}^{N} \frac{m_{t}}{\Omega_{t}}\right|_{m_{1}, \ldots, m_{N}, 0} ^{1}\right]\right)\right)
\end{aligned}
$$

\section{The OP of the Suboptimal TAS Scheme}

\section{1. $\gamma_{t h}>\gamma_{P}$}

If $\gamma_{\text {th }}>\gamma_{\mathrm{P}}$, the OP of the suboptimal TAS scheme can be expressed as

$$
\begin{aligned}
F_{\text {suboptimal }} & =\operatorname{Pr}\left(\gamma_{\mathrm{p}}<\gamma_{\mathrm{SD} g}<\gamma_{\mathrm{th}}\right)+\operatorname{Pr}\left(\gamma_{\mathrm{SD} g}<\gamma_{\mathrm{p}}, \gamma_{\mathrm{SR}}<\gamma_{\mathrm{T}}\right)+\operatorname{Pr}\left(\gamma_{\mathrm{SD} g}<\gamma_{\mathrm{p}}, \gamma_{\mathrm{SR}}>\gamma_{\mathrm{T}}, \gamma_{\mathrm{RD}}<\gamma_{\mathrm{th}}\right) \\
& =G G_{1}+G G_{2}+G G_{3}
\end{aligned}
$$

where

$$
\gamma_{\mathrm{SD} g}=\max _{1 \leqslant i \leqslant N_{t}, 1 \leqslant j \leqslant N_{r}}\left(\gamma_{\mathrm{SD} i j}\right)
$$

$G G_{1}$ is given by

$$
\begin{aligned}
& G G_{1}=\operatorname{Pr}\left(\gamma_{\mathrm{p}}<\gamma_{\mathrm{SD} g}<\gamma_{\text {th }}\right) \\
& =\left(\frac{1}{\prod_{d=1}^{N} \Gamma\left(m_{d}\right)} G_{1, N+1}^{N, 1}\left[\left.\frac{\gamma_{\text {th }}}{\gamma_{\mathrm{SD}}} \prod_{d=1}^{N} \frac{m_{d}}{\Omega_{d}}\right|_{m_{1}, \ldots, m_{N}, 0} ^{1}\right]\right)^{N_{t} \times N_{r}}-\left(\frac{1}{\prod_{d=1}^{N} \Gamma\left(m_{d}\right)} G_{1, N+1}^{N, 1}\left[\left.\frac{\gamma_{\mathrm{P}}}{\gamma_{\mathrm{SD}}} \prod_{d=1}^{N}\right|_{m_{1}, \ldots, m_{N}, 0} ^{1}\right]\right)^{N_{t} \times N_{r}}
\end{aligned}
$$

$G G_{2}$ can be written as

$$
\begin{aligned}
& G G_{2}=\operatorname{Pr}\left(\gamma_{\mathrm{SD} g}<\gamma_{\mathrm{p}}, \gamma_{\mathrm{SR}}<\gamma_{\mathrm{T}}\right) \\
& =\left(\frac{1}{\prod_{d=1}^{N} \Gamma\left(m_{d}\right)} G_{1, N+1}^{N, 1}\left[\left.\frac{\gamma_{\mathrm{P}}}{\overline{\gamma_{\mathrm{SD}}}} \prod_{d=1}^{N} \frac{m_{d}}{\Omega_{d}}\right|_{m_{1}, \ldots, m_{N}, 0} ^{1}\right]\right)^{N_{t} \times N_{r}} \times\left(\frac{1}{\prod_{t=1}^{N} \Gamma\left(m_{t}\right)} G_{1, N+1}^{N, 1}\left[\left.\frac{\gamma_{\mathrm{T}}}{\bar{\gamma}_{\mathrm{SR}}} \prod_{t=1}^{N} \frac{m_{t}}{\Omega_{t}}\right|_{m_{1}, \ldots, m_{N}, 0} ^{1}\right]\right)^{L}
\end{aligned}
$$


and $G G_{3}$ can be expressed as

$$
\begin{aligned}
& G G_{3}=\operatorname{Pr}\left(\gamma_{\mathrm{SD} g}<\gamma_{\mathrm{p}}, \gamma_{\mathrm{SR}}>\gamma_{\mathrm{T}}, \gamma_{\mathrm{RD}}<\gamma_{\mathrm{th}}\right) \\
& =\left(\frac{1}{\prod_{d=1}^{N} \Gamma\left(m_{d}\right)} G_{1, N+1}^{N, 1}\left[\left.\frac{\gamma_{\mathrm{P}}}{\bar{\gamma}_{\mathrm{SD}}} \prod_{d=1}^{N} \frac{m_{d}}{\Omega_{d}}\right|_{m_{1}, \ldots, m_{N}, 0} ^{1}\right]\right)^{N_{t} \times N_{r}} \times \frac{1}{\prod_{t t=1}^{N} \Gamma\left(m_{t t}\right)} G_{1, N+1}^{N, 1}\left[\left.\frac{\gamma_{\mathrm{th}}}{\bar{\gamma}_{\mathrm{RD}}} \prod_{t t=1}^{N} \frac{m_{t t}}{\Omega_{t t}}\right|_{m_{1}, \ldots, m_{N}, 0} ^{1}\right] \times \\
& \left(1-\left(\frac{1}{\prod_{t=1}^{N} \Gamma\left(m_{t}\right)} G_{1, N+1}^{N, 1}\left[\left.\frac{\gamma_{\mathrm{T}}}{\gamma_{\mathrm{SR}}} \prod_{t=1}^{N} \frac{m_{t}}{\Omega_{t}}\right|_{m_{1}, \ldots, m_{N}, 0} ^{1}\right]\right)\right)
\end{aligned}
$$

4.2. $\gamma_{t h}<\gamma_{P}$

If $\gamma_{\text {th }}<\gamma_{\mathrm{P}}$, the OP of the suboptimal TAS scheme can be expressed as

$$
\begin{aligned}
F_{\text {suboptimal }} & =\operatorname{Pr}\left(\gamma_{\mathrm{SD} g}<\gamma_{\text {th }}, \gamma_{\mathrm{SR}}<\gamma_{\mathrm{T}}\right)+\operatorname{Pr}\left(\gamma_{\mathrm{SD} g}<\gamma_{\text {th }}, \gamma_{\mathrm{SR}}>\gamma_{\mathrm{T}}, \gamma_{\mathrm{RD}}<\gamma_{\text {th }}\right) \\
& =G G_{11}+G G_{22}
\end{aligned}
$$

where $G G_{11}$ can be written as

$$
\begin{aligned}
& G G_{11}=\operatorname{Pr}\left(\gamma_{\mathrm{SD} g}<\gamma_{\mathrm{th}}, \gamma_{\mathrm{SR}}<\gamma_{\mathrm{T}}\right) \\
& =\left(\frac{1}{\prod_{d=1}^{N} \Gamma\left(m_{d}\right)} G_{1, N+1}^{N, 1}\left[\left.\frac{\gamma_{\mathrm{th}}}{\overline{\gamma_{\mathrm{SD}}}} \prod_{d=1}^{N} \frac{m_{d}}{\Omega_{d}}\right|_{m_{1}, \ldots, m_{N}, 0} ^{1}\right]\right)^{N_{t} \times N_{r}} \times\left(\frac{1}{\prod_{t=1}^{N} \Gamma\left(m_{t}\right)} G_{1, N+1}^{N, 1}\left[\left.\frac{\gamma_{T}}{\gamma_{\mathrm{SR}}} \prod_{t=1}^{N} \frac{m_{t}}{\Omega_{t}}\right|_{m_{1}, \ldots, m_{N}, 0} ^{1}\right]\right)^{L}
\end{aligned}
$$

and $G G_{22}$ can be expressed as

$$
\begin{aligned}
& G G_{22}=\operatorname{Pr}\left(\gamma_{\mathrm{SD} g}<\gamma_{\mathrm{th}}, \gamma_{\mathrm{SR}}>\gamma_{\mathrm{T}}, \gamma_{\mathrm{RD}}<\gamma_{\mathrm{th}}\right) \\
& =\left(\frac{1}{\prod_{d=1}^{N} \Gamma\left(m_{d}\right)} G_{1, N+1}^{N, 1}\left[\left.\frac{\gamma_{\mathrm{th}}}{\bar{\gamma}_{\mathrm{SD}}} \prod_{d=1}^{N} \frac{m_{d}}{\Omega_{d}}\right|_{m_{1}, \ldots, m_{N}, 0} ^{1}\right]\right) \times \frac{1}{\prod_{t t=1}^{N} \Gamma\left(m_{t t}\right)} G_{1, N+1}^{N, 1}\left[\left.\frac{\gamma_{\mathrm{th}}}{\bar{\gamma}_{\mathrm{RD}}} \prod_{t t=1}^{N} \frac{m_{t t}}{\Omega_{t t}}\right|_{m_{1}, \ldots, m_{N}, 0}\right] \times \\
& \left(1-\left(\frac{1}{\prod_{t=1}^{N} \Gamma\left(m_{t}\right)} G_{1, N+1}^{N, 1}\left[\left.\frac{\gamma_{\mathrm{T}}}{\bar{\gamma}_{\mathrm{SR}}} \prod_{t=1}^{N} \frac{m_{t}}{\Omega_{t}}\right|_{m_{1}, \ldots, m_{N}, 0} ^{1}\right]\right)\right)
\end{aligned}
$$

\section{Optimal Power Allocation}

Figure 2 presents the effect of the power allocation parameter $K$ on the OP performance. The parameters are $N=2, m=2, \mu=0 \mathrm{~dB}, N_{t}=2, L=2, N_{r}=2, \gamma_{\text {th }}=5 \mathrm{~dB}, \gamma_{\mathrm{T}}=2 \mathrm{~dB}$, and $\gamma_{\mathrm{P}}=2 \mathrm{~dB}$. These results show that the OP performance improves as the SNR is increased. For example, when $K=0.7$, the OP is $2.3 \times 10^{-2}$ with SNR $=10 \mathrm{~dB}, 1.3 \times 10^{-4}$ with SNR $=15 \mathrm{~dB}$, and $2.4 \times 10^{-7}$ with $\mathrm{SNR}=20 \mathrm{~dB}$. The optimum value of $K$ is 0.86 with $\mathrm{SNR}=10 \mathrm{~dB}, 0.92$ with $\mathrm{SNR}=15 \mathrm{~dB}$, and 0.96 with $\mathrm{SNR}=20 \mathrm{~dB}$. This indicates that equal power allocation (EPA) is not the best scheme.

Unfortunately, it is very difficult to derive a closed-form expression for $K$. Because the OP expressions are very complex, numerical methods are used to solve the optimization problem. The optimum power allocation (OPA) values were obtained for given values of SNR and system parameters. Table 1 presents the optimum values of $K$ for three values of relative geometrical gain $\mu=5 \mathrm{~dB}, 0 \mathrm{~dB},-5 \mathrm{~dB}$. The other parameters are $N=2, m=2, N_{t}=2, L=2, \gamma_{\text {th }}=5 \mathrm{~dB}, \gamma_{\mathrm{T}}=2 \mathrm{~dB}$, and $\gamma_{\mathrm{P}}=6 \mathrm{~dB}$. For example, with a low SNR and $\mu=5 \mathrm{~dB}$, nearly all of the power should be used in the broadcast phase. As the SNR increases, the optimum value of $K$ is reduced, so that at $\mathrm{SNR}=20 \mathrm{~dB}$ only half of the power should be used in the broadcast phase. 


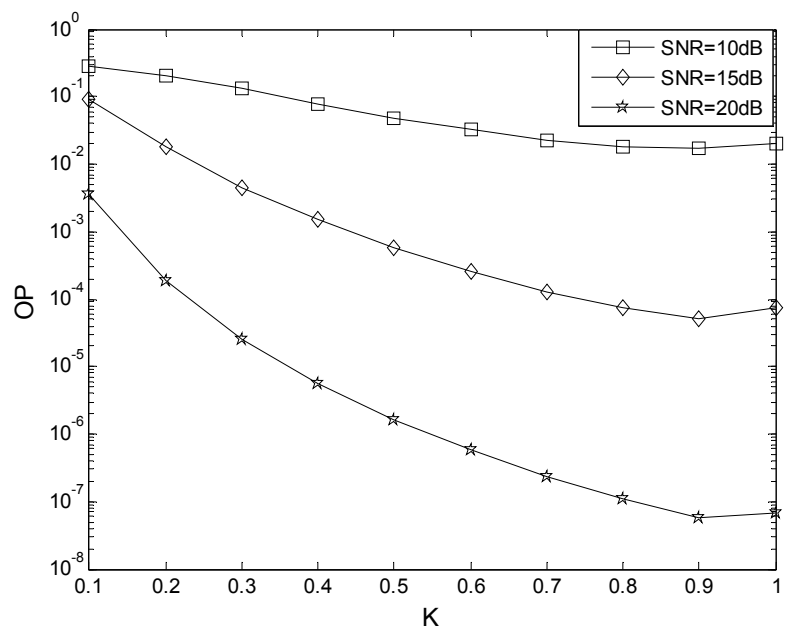

Figure 2. The effect of the power allocation parameter $K$ on the OP performance.

Table 1. OPA parameters $K$.

\begin{tabular}{cccc}
\hline SNR (dB) & $\boldsymbol{\mu}=\mathbf{5} \mathbf{d B}$ & $\boldsymbol{\mu}=\mathbf{0} \mathbf{d B}$ & $\boldsymbol{\mu}=-\mathbf{5} \mathbf{d B}$ \\
\hline 5 & 0.99 & 0.41 & 0.51 \\
10 & 0.51 & 0.41 & 0.47 \\
15 & 0.50 & 0.44 & 0.45 \\
20 & 0.50 & 0.47 & 0.46 \\
\hline
\end{tabular}

Figure 3 presents the effect of the relative geometrical gain $\mu$ on the OP performance using the values of $K$ given in Table 1 . These results show that the OP performance improves as $\mu$ decreases. For example, when $\mathrm{SNR}=10 \mathrm{~dB}$, the $\mathrm{OP}$ is $3.6 \times 10^{-3}$ for $\mu=5 \mathrm{~dB}, 4.5 \times 10^{-5}$ for $\mu=0 \mathrm{~dB}$, and $1.9 \times 10^{-7}$ for $\mu=-5 \mathrm{~dB}$. This indicates that the best location for the relay is near the destination. For fixed $\mu$, an increase in the SNR reduces the OP, as expected.

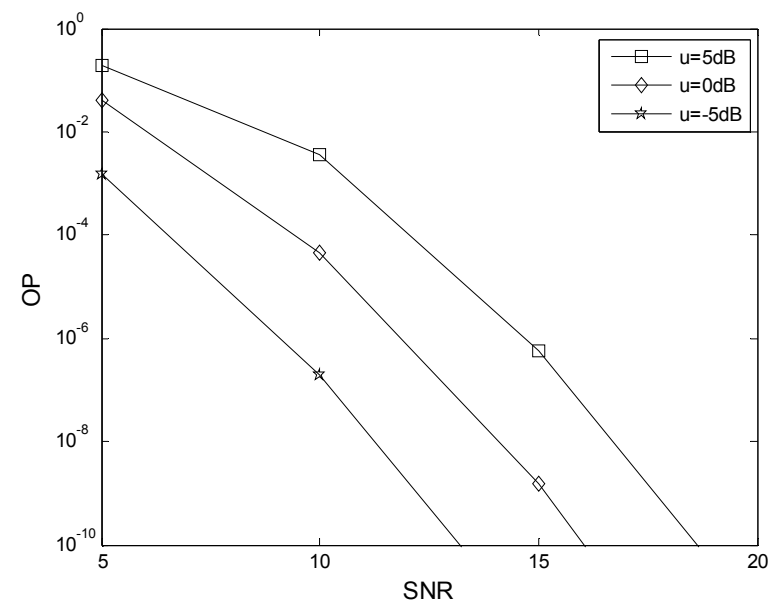

Figure 3. The effect of the relative geometrical gain $\mu$ on the OP performance.

\section{Numerical Results}

In this section, simulation results are presented to confirm the analysis given previously. The Monte Carlo simulations were done using MATLAB, and the analytical results were verified using MAPLE. The total energy is $E=1$, the fading coefficient is $m=1,2,3$, the number of cascaded 
components is $N=2,3,4$, the number of mobile relays is $L=2$, the number of receive antennas is $N_{r}=2$, the relative geometrical gain is $\mu=0 \mathrm{~dB}$, and the number of transmit antennas is $N_{t}=1,2,3$.

Figures 4 and 5 present the OP performance of the optimal TAS scheme with $\gamma_{\mathrm{th}}=5 \mathrm{~dB}, \gamma_{\mathrm{T}}=2 \mathrm{~dB}$ and $\gamma_{\mathrm{P}}=6 \mathrm{~dB}$, and $\gamma_{\mathrm{th}}=5 \mathrm{~dB}, \gamma_{\mathrm{T}}=2 \mathrm{~dB}$ and $\gamma_{\mathrm{P}}=3 \mathrm{~dB}$, respectively. The other parameters are $N=2$, $m=2, K=0.5, N_{t}=1,2,3, L=2, N_{r}=2$, and $\mu=0 \mathrm{~dB}$. This shows that the analytical results match the simulation results. The OP improves as the number of transmit antennas is increased. For example, when $\gamma_{\mathrm{th}}=5 \mathrm{~dB}, \gamma_{\mathrm{T}}=2 \mathrm{~dB}, \gamma_{\mathrm{P}}=3 \mathrm{~dB}$, and SNR $=10 \mathrm{~dB}$, the OP is $1.1 \times 10^{-1}$ when $N_{t}=1,1.3 \times 10^{-2}$ when $N_{t}=3$, and $1.4 \times 10^{-3}$ when $N_{t}=3$. For fixed $N_{t}$, an increase in the SNR decreases the OP.

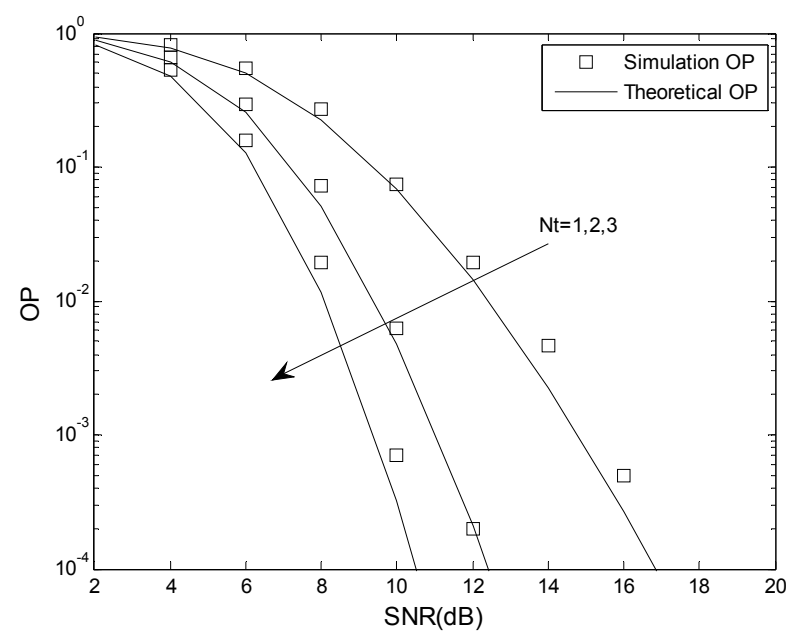

Figure 4. The OP performance of the optimal TAS scheme when $\gamma_{\mathrm{th}}<\gamma_{\mathrm{P}}$.

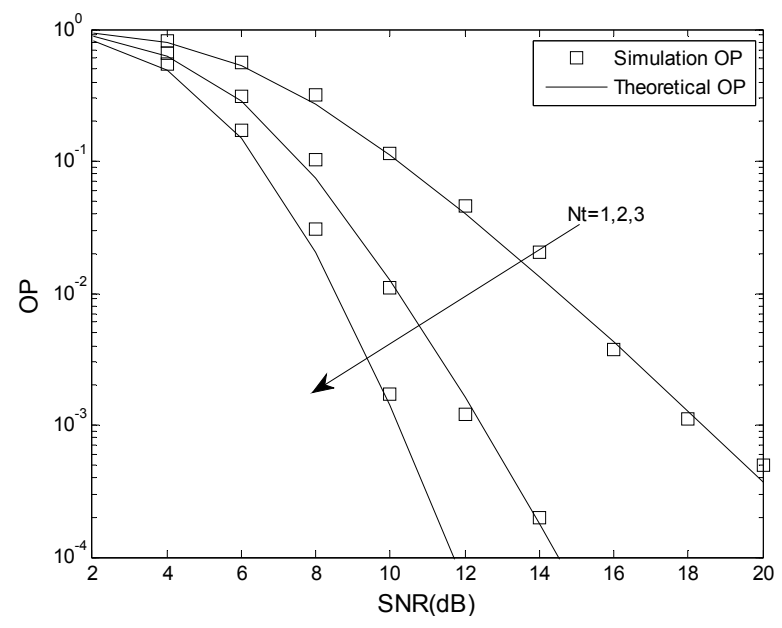

Figure 5. The OP performance of the optimal TAS scheme when $\gamma_{\text {th }}>\gamma_{\mathrm{P}}$.

Figures 6 and 7 present the OP performance of the suboptimal TAS scheme with $\gamma_{\text {th }}=5 \mathrm{~dB}$, $\gamma_{\mathrm{T}}=2 \mathrm{~dB}$ and $\gamma_{\mathrm{P}}=6 \mathrm{~dB}$, and $\gamma_{\mathrm{th}}=5 \mathrm{~dB}, \gamma_{\mathrm{T}}=2 \mathrm{~dB}$ and $\gamma_{\mathrm{P}}=3 \mathrm{~dB}$, respectively. The other parameters are $N=2, m=2, K=0.5, N_{t}=1,2,3, L=2, N_{r}=2$, and $\mu=0 \mathrm{~dB}$. This also shows that the analytical results match the simulation results. As expected, the OP improves as the number of transmit antennas is increased. For example, when $\gamma_{\mathrm{th}}=5 \mathrm{~dB}, \gamma_{\mathrm{T}}=2 \mathrm{~dB}, \gamma_{\mathrm{P}}=6 \mathrm{~dB}, \mathrm{SNR}=12 \mathrm{~dB}$, and $N_{t}=1$, the OP is $4.1 \times 10^{-2}$ when $N_{t}=1,4.7 \times 10^{-3}$ when $N_{t}=2$, and $5.3 \times 10^{-4}$ when $N_{t}=3$, For fixed $N_{t}$, an increase in the SNR decreases the OP, as expected. 


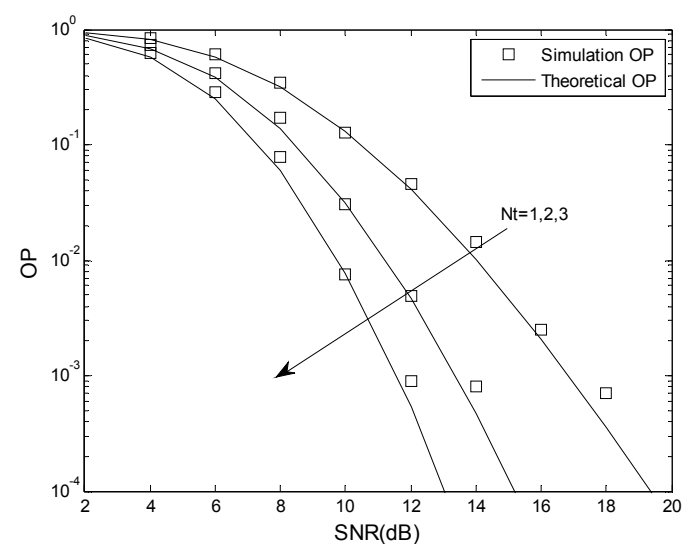

Figure 6. The OP performance of the suboptimal TAS scheme when $\gamma_{\text {th }}<\gamma_{\mathrm{P}}$.

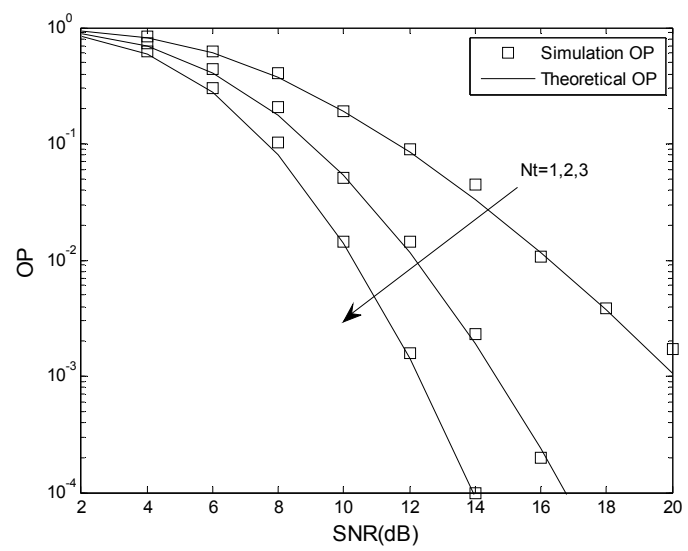

Figure 7. The OP performance of the suboptimal TAS scheme when $\gamma_{\mathrm{th}}>\gamma_{\mathrm{P}}$.

Figure 8 compares the OP performance of the optimal and suboptimal TAS schemes for different numbers of antennas $N_{t}$. The parameters are $N=2, m=2, K=0.5, \mu=0 \mathrm{~dB}, N_{t}=2,3, L=2, N_{r}=2$, $\gamma_{\mathrm{th}}=5 \mathrm{~dB}, \gamma_{\mathrm{T}}=2 \mathrm{~dB}$, and $\gamma_{\mathrm{P}}=3 \mathrm{~dB}$. In all cases, for a given value of $N_{t}$ the optimal TAS scheme has better OP performance. As predicted by the analysis, the performance gap between the two TAS schemes decreases as $N_{t}$ is increased. When the SNR is low, the OP performance gap between the optimal TAS scheme with $N_{t}=2$ and the suboptimal TAS scheme with $N_{t}=3$ is negligible. As the SNR increases, the OP performance gap also increases.

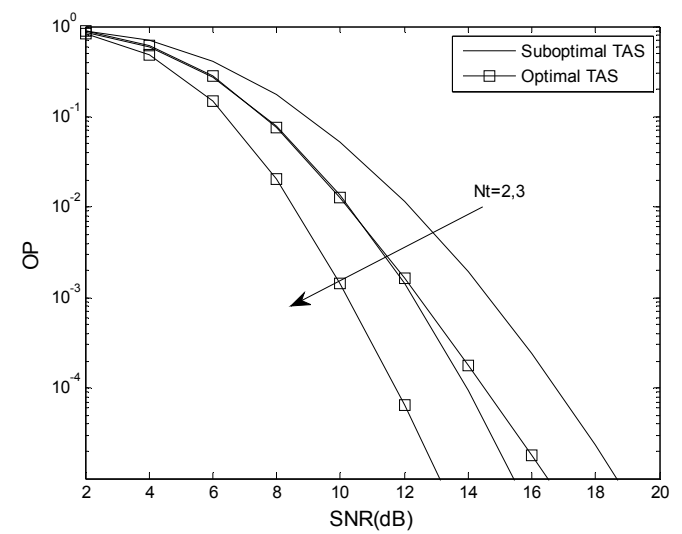

Figure 8. The OP performance of the optimal and suboptimal TAS schemes for different numbers of antennas $N_{t}$. 


\section{Conclusions}

In this paper, exact closed-form OP expressions were derived for ISDF relaying M2M networks with TAS over N-Nakagami fading channels. Performance results were presented which show that the optimal TAS scheme has better OP performance than the suboptimal scheme. It was also shown that the power allocation parameter $K$ can have a significant effect on the OP performance. The given expressions can be used to evaluate the OP performance of inter-vehicular networks, mobile wireless sensor networks, and mobile heterogeneous networks.

Acknowledgments: The authors would like to thank the referees and editors for providing very helpful comments and suggestions. This project was supported by the National Natural Science Foundation of China (Nos. 61304222 and 61301139), and the Shandong Province Outstanding Young Scientist Award Fund (No. 2014BSE28032).

Author Contributions: The corresponding author Lingwei Xu derived the exact closed-form OP expressions for the optimal and suboptimal TAS schemes; Hao Zhang conceived and designed the experiments; T. Aaron Gulliver provided comments on the paper organization, contributed towards the performance results and analytic evaluations, and was involved in the creation of the manuscript.

Conflicts of Interest: The authors declare no conflicts of interest.

\section{References}

1. Wu, G.; Talwar, S.; Johnsson, K.; Himayat, N.; Johnson, K.D. M2M: From mobile to embedded internet. IEEE Commun. Mag. 2011, 49, 36-43.

2. Chen, M.; Wan, J.F.; Li, F. Machine-to-machine communications: Architectures, standards, and applications. KSII Trans. Int. Inf. Syst. 2012, 6, 480-497. [CrossRef]

3. Jo, M.; Maksymyuk, T.; Strykhalyuk, B.; Cho, C.H. Device-to-device based heterogeneous radio access network architecture for mobile cloud computing. IEEE Wirel. Commun. 2015, 22, 50-58. [CrossRef]

4. Mumtaz, S.; Huq, K.M.S.; Rodriguez, J. Direct mobile-to-mobile communication: Paradigm for 5G. IEEE Wirel. Commun. 2014, 21, 14-23. [CrossRef]

5. Chen, M.; Wan, J.F.; Gonzalez, S.; Liao, X.F.; Leung, V.C.M. A survey of recent developments in home M2M networks. IEEE Commun. Surv. Tutor. 2014, 16, 98-114. [CrossRef]

6. Talha, B.; Patzold, M. Channel models for mobile-to-mobile cooperative communication systems: A state of the art review. IEEE Veh. Technol. Mag. 2011, 6, 33-43. [CrossRef]

7. Salo, J.; Sallabi, H.E.; Vainikainen, P. Statistical analysis of the multiple scattering radio channel. IEEE Trans. Antennas Propag. 2006, 54, 3114-3124. [CrossRef]

8. Salo, J.; Sallabi, H.E.; Vainikainen, P. The distribution of the product of independent Rayleigh random variables. IEEE Trans. Antennas Propag. 2006, 54, 639-643. [CrossRef]

9. Uysal, M. Diversity analysis of space-time coding in cascaded Rayleigh fading channels. IEEE Commun. Lett. 2006, 10, 165-167. [CrossRef]

10. Karagiannidis, G.K.; Sagias, N.C.; Mathiopoulos, P.T. N-Nakagami: A novel stochastic model for cascaded fading channels. IEEE Trans. Commun. 2007, 55, 1453-1458. [CrossRef]

11. Gong, F.K.; Ye, P.; Wang, Y.; Zhang, N. Cooperative mobile-to-mobile communications over double Nakagami-m fading channels. IET Commun. 2012, 6, 3165-3175. [CrossRef]

12. Ilhan, H.; Uysal, M.; Altunbas, I. Cooperative diversity for intervehicular communication: Performance analysis and optimization. IEEE Trans. Veh. Technol. 2009, 58, 3301-3310. [CrossRef]

13. Xu, L.W.; Zhang, H.; Liu, X.; Gulliver, T.A. Performance analysis of FAF relaying M2M cooperative networks over N-Nakagami fading channels. Int. J. Signal Process. Image Process. Pattern Recognit. 2015, 8, $249-258$. [CrossRef]

14. Xu, L.W.; Zhang, H.; Lu, T.T.; Liu, X.; Wei, Z.Q. Performance analysis of the mobile-relay-based M2M communication over N-Nakagami fading channels. J. Appl. Sci. Eng. 2015, 18, 309-314.

15. Ikki, S.S.; Ahmed, M.H. Performance analysis of incremental-relaying cooperative-diversity networks over Rayleigh fading channels. IET Commun. 2011, 5, 337-349. [CrossRef]

16. Yang, C.; Wang, W.; Chen, S.; Peng, M. Outage performance of orthogonal space-time block codes transmission in opportunistic decode-and-forward cooperative networks with incremental relaying. IET Commun. 2011, 5, 61-70. [CrossRef] 
17. Xu, L.W.; Zhang, H.; Lu, T.T.; Gulliver, T.A. Performance analysis of the IAF relaying M2M cooperative networks over N-Nakagami fading channels. J. Commun. 2015, 10, 185-191. [CrossRef]

18. Xu, L.W.; Zhang, H.; Gulliver, T.A. Performance analysis of IDF relaying M2M cooperative networks over N-Nakagami fading channels. KSII Trans. Int. Inf. Syst. 2015, 9, 3983-4001.

19. Chen, H.; Liu, J.; Zhai, C.; Liu, Y.X. Performance of incremental-selective decode-and-forward relaying cooperative communications over Rayleigh fading channels. In Proceedings of the Conference on Wireless Communications \& Signal Processing, Nanjing, China, 13-15 November 2009; pp. 1-5.

20. Xu, L.W.; Zhang, H.; Gulliver, T.A. Performance analysis of the SIR M2M cooperative networks. Int. J. Control Autom. 2015, 8, 407-416. [CrossRef]

21. Suraweera, H.A.; Smithnd, P.J.; Nallanathan, A.; Thompson, J.S. Amplify-and-forward relaying with optimal and suboptimal transmit antenna selection. IEEE Trans. Wirel. Commun. 2011, 10, 1874-1885. [CrossRef]

22. Yeoh, P.L.; Elkashlan, M.; Yang, N.; Costa, D.B.D.; Duong, T.Q. Unified analysis of transmit antenna selection in MIMO multi-relay networks. IEEE Trans. Veh. Technol. 2013, 62, 933-939. [CrossRef]

23. Li, H.; Song, L.Y.; Debbah, M. Energy efficiency of large-scale multiple antenna systems with transmit antenna selection. IEEE Trans. Commun. 2014, 62, 638-647. [CrossRef]

24. Zhang, H.J.; Jiang, C.X.; Beaulieu, N.C.; Chu, X.L.; Wen, X.M.; Tao, M.X. Resource Allocation in Spectrum-Sharing OFDMA Femtocells with Heterogeneous Services. IEEE Trans. Commun. 2014, 62, 2366-2377. [CrossRef]

25. Zhang, H.J.; Jiang, C.X.; Beaulieu, N.C.; Chu, X.L.; Wang, X.B.; Quek, T.Q.S. Resource Allocation for Cognitive Small Cell Networks: A Cooperative Bargaining Game Theoretic Approach. IEEE Trans. Wirel. Commun. 2015, 14, 3481-3493. [CrossRef]

26. Zhang, H.J.; Jiang, C.X.; Mao, X.T.; Chen, H.H. Interference-Limit Resource Optimization in Cognitive Femtocells with Fairness and Imperfect Spectrum Sensing. IEEE Trans. Veh. Technol. 2015. [CrossRef]

27. Zhang, H.J.; Xing, H.; Cheng, J.L.; Nallanathan, A.; Leung, V.C.M. Secure Resource Allocation for OFDMA Two-Way Relay Wireless Sensor Networks Without and With Cooperative Jamming. IEEE Trans. Ind. Inform. 2015. [CrossRef]

28. Ochiai, H.; Mitran, P.; Tarokh, V. Variable-rate two-phase collaborative communication protocols for wireless networks. IEEE Trans. Veh. Technol. 2006, 52, 4299-4313. [CrossRef]

(C) 2016 by the authors; licensee MDPI, Basel, Switzerland. This article is an open access article distributed under the terms and conditions of the Creative Commons by Attribution (CC-BY) license (http:/ / creativecommons.org/licenses/by/4.0/). 